\title{
ANAESTHETIC MANAGEMENT OF A ECTOPIC PREGNANCY PATIENT WITH ORGANOPHOSPHOROUS POISONING
}

\author{
N. Gopal Reddy¹, Kahlekar², Laxmi Priyanka³
}

\section{HOW TO CITE THIS ARTICLE:}

N. Gopal Reddy, Kahlekar, Laxmi Priyanka. "Anaesthetic Management of a Ectopic Pregnancy Patient with Organophosphorous Poisoning". Journal of Evolution of Medical and Dental Sciences 2015; Vol. 4, Issue 02, January 05; Page: 285-296, DOI: 10.14260/jemds/2015/45

ABSTRACT: Organophosphorus poisoning is the most common poisoning in India because of its easy availability. Anaesthesiologists may come across rarely to give anaesthesia for emergency life-saving surgeries. Problems while anaesthetising an emergency patient following organophosphorous poisoning are many. Necessary precautions are to be taken while anaesthetising the patient. During anaesthesia many drugs are used which are linked with acetylcholine, cholinesterase and other cardio-vascular effects.

KEYWORDS: acetylcholine, cholinergic crisis, intermediate syndrome, organophosphate insecticide poisoning, ruptured ectopic pregnancy, emergency operation.

INTRODUCTION: Organophosphorus compounds, used as insecticides and agents of chemical warfare, are a major Global cause of health problems. These irreversible inhibitors of cholinesterase produce three wells-recognised clinical entities: the initial cholinergic phase, which is a medical emergency often requiring management in an intensive care unit; the intermediate syndrome, during which prolonged ventilatory care is necessary; and delayed polyneuropathy. In addition, disturbances of body temperature and endocrine function, electrolyte imbalances, immunological dysfunction and disorders of reproduction have been reported. Vocal cord paralysis, pancreatitis, cardiac arrhythmias and a wide range of neuropsychiatric disorders are known to follow acute and chronic exposure to organophosphorus compounds.

As a result of the inhibition of plasma cholinesterase, there can be increased sensitivity to drugs hydrolysed by this enzyme, e.g. suxamethonium and mivacurium. The inhibition of acetycholinesterase causes dysfunction at the neuromuscular junction which can produce altered responses to non-depolarizing neuromuscular blockers.

Anaesthetists may encounter patients exposed to organophosphorus compounds either following acute poisoning, trauma (warfare) or as patients with a wide range of nonspecific disorders presenting for surgery.

CASE REPORT: 22yrs unmarried female has consumed unknown quantity of organophosphorous (chlorpyrifos) compound around $10 \mathrm{pm}$ on $17 / 10 / 14$. She was brought to the hospital by her mother at about 6.40 am on 18/10/14. Patient presented to casualty with symptoms of sweating, salivation, Lacrimation, headache, nausea and vomiting. Patient had 1 episode of vomiting. She was diagnosed outside to have ectopic pregnancy and thus had consumed organophosphorus poison. Patient was conscious, altered sensorium and irritable.

VITAL SIGNS: Glasgow coma scale (GCS)- 12/15, Temperature- $102 * \mathrm{~F}$, 02 Saturation- $92 \%$ on room air, 94\% with Fio2 0.4,Pulse rate(P. R) 108 bpm, Blood Pressure 100/70 mm Hg, Respiratory Rate 28 


\section{CASE REPORT}

pm. Pupils were bilaterally constricted, profuse oral secretions, profuse sweating, garlic like odour breath and Pallor ++.

LUNGS: Respiratory pattern-normal, bilateral harsh vesicular breath sounds, crepitations heard in both lungs in lower zones, no wheeze.

NEUROMUSCULAR: Fasiculations + ve, occasional jerky movements+ve.

OTHERS: vaginal bleeding+, extremities-cool but not cyanotic, no edema.

As blood gas analysis within normal limits no active ventilatory intervention was required. Mechanical ventilator kept standby.

GYNAECOLOGY REFERRAL WAS EXTENDED: per abdomen-soft, tenderness in right iliac fossa, per Vaginal bleeding+. Vaginal examination-cervical motion tenderness+, right fornix tenderness+, cervix deviated to left.

Provisional diagnosis of organophosphorus poisoning with ectopic pregnancy was made and treated in the same guidelines.

Plan initiated to stabilize the patient and planed for emergency laparotomy once the patient is stabilized.

\section{TREATMENT OF O.P POISONING:}

1. Decontamination of patient was done Nasogastric tube $18 \mathrm{FG}$ was inserted, gastric lavage with 2liters of normal saline was done until clear fluid aspirate was obtained. 2. Two 18 G I.V cannulas were secured to both hands. 3. Inj. Atropine $4 \mathrm{mg}$ I.V stat followed by $8 \mathrm{mcg} / \mathrm{kg} / \mathrm{min}$ continuous infusion till full atropinisation. Then $2 \mathrm{mg}$ I.V hrly was continued. 4 . Inj. PAM test dose $1 \mathrm{gm} \mathrm{I.V} \mathrm{infusion} \mathrm{followed} \mathrm{by} \mathrm{loading} \mathrm{dose} 2 \mathrm{gm}$ over hrs in 1 pint NS, followed by $6 \mathrm{gm}$ over 12 hrs BD was administered. 5. IVF-DNS, RL, NS @ U.0/hr+50ml/hr. 6. Inj. Pantop 40mg O.D, I.V. 7. Inj. Monocef 1gm B.D, I.V. 8. Inj. Ondensetron 4mg B.D, I.V. 9. Foleys catheterisation. Vitals were monitored, input output charting hrly was done.

INVESTIGATIONS: ABG-(on room air). PH-7.40,Pco2-37,Po2-83,HCO3-22.4,BEB 4.8,02sat-90\%, CBP$\mathrm{Hb}-7.6 \mathrm{gm} \%$, Hct-22\%, Platelet count 3lks/mm3, COAGULATION PROFILE: BT- 2min, CT-4min; PT16sec, INR-1.2, APTT-31sec, BLOOD GROUP: 0+ve, LFTs-WNL, Total proteins -5.9gm/dl(low), RFTsWNL

SPECIAL INVESTIGATIONS: Pregnancy Test- weakly positive.

USG abdomen and pelvis-(bedside): free fluid in the pouch of douglas. ascites in the right iliac fossa, Sr. pseudocholinesterase-391 u/l (low).

CXR- wnl, 12lead ECG-sinus tachycardia.

Planed for emergency laparotomy, once the patient was stabilized, pre anaesthetic checkup was done. 3 units packed red cells, 2 units FFPs reserved.NBM status was continued.

Aspiration prophylaxis-inj.ondensetron, inj.pantop was given. 
Patient was shifted @2.30pm General anaesthesia was Planned: Patient was connected to multiparamonitor and vitals noted. Pulse Rate-130/ min, low volume. Blood Pressure- 90/60mmHg, Spo 2-94\% (On room air), R.R- 27/min. Shifted to O.T with 1 unit packed cell I.V infusion running. IVF - RL was started on the other hand@ $50 \mathrm{ml} / \mathrm{hr}$. Urine output- $150 \mathrm{ml}$ was noted. Nasogartric tube suction was adequately done.

Patient was preoxgenated for 3min with 100\% o2 @ 8l/min.

PREMEDICATION: Inj. Fentanyl 50 mcg i.v was given, Induction- inj. Midazolam3 mg i.v.

INTUBATION: inj. Vecuronium 3mg i. v. Patient was intubated with $7.0 \mathrm{~mm}$ cuffed ETT and after checking for B/L air entry, ETT was fixed @ $18 \mathrm{~cm}$ mark and connected to Boyles machine with bains coaxial circuit. Maintenance- N20:02= 4:4 l/min, Intraoperative hemodynamics were stable. Top up doses with atricurium twice given;

1 unit packed cells was transfused. Blood loss- 500ml (hemoperitoneum noted).Ruptured ectopic rt. was noted, excised and fallopian tube was ligated. Haemostasis was secured. U.O- 00ml, clear.

Hemodynamics were stable at the end of the surgery. Surgery was completed @ 3.45 pm.

Patient was planned on elective mechanical ventilation Post-operatively: Ventilator settings: mode-IPPV, T.V-350ml, freq-14/min, Fio2-0.7, peep-3cm H2o. Inj. vecuronium 1mg and inj. Midazolam $1 \mathrm{mg}$ hrly. Patient was weaned from IPPV $\rightarrow$ SIMV mode.

$2^{\text {nd }}$ unit packed cells and 1 unit FFP were continued postoperatively.

Inj. Atropine and PAM were continued. Antibotics-inj. Piptaz 4.5 gm i.v bd, Inj.metrogyl 100ml i.v tid, U.O- $50 \mathrm{ml} / \mathrm{hr}$, clear, $2^{\text {nd }}$ unit FFP was transfused.

Central line placed (rt. Subclavian vein), $\mathrm{CVP}=8 \mathrm{cmH} 20$,

19/10/14 (Post op day1-Investigations): ABG: PH-7.42, PCO2-27, P02-94, HCO3-17.2, BEB-5.2, 02 sat-97.6 $\mathrm{Hb}=8.3 \mathrm{gm} \%$, Hct=24.3\%, Coagulation profile-wnl, CXR- WNL.

Patient c/o tingling sensation and spasms of both hands, Sr. electrolytes: Na $146 \mathrm{mmol} / \mathrm{l}, \mathrm{K}$ 3mmol/l, Ca 7.9mmol/l

Physician referral done: Inj.calcium gluconate 1gm slow i.v over 10min, Syr.potchlor bd through Ryles tube.

20/10/14 (Oostop day 2): Patient respiratory efforts were good, Chest -clear, Well conscious and coherent. Weaned to CPAP and alternated with T-piece. haemodynamically stable.

5.30pm: After fulfilling extubation criteria, adequate nebulisation with duolin and budecort given, inj.hydrocortisone i.v, adequate chest physiotherapy, oral suctioning, patient was extubatubated. 02 inhalation by facemask@ 6l/min for 8hrs, Propped up position. Adviced ABG after 2hrs: ph=7.37, pco2 $=33$, pa02=201, HCO3=18.6, 02 sat=99.7. Sr. pseudo cholinesterase $=1890 \mathrm{u} / \mathrm{l}, \mathrm{CXR}-\mathrm{wnl}$

21/10/14 (Post extubation day 1): 4pmIn view of altered GCS, falling spo $2=60 \%$.

R.R=30/min, rapid shallow breathing, inadequate respiratory efforts, patient was reintubated and connected to mechanical ventilator.

ABG-Implied primary uncompensated respiratory alkalosis. 


\section{CASE REPORT}

Chest: B/L air entry +, coarse crepts all over chest.

Diagnosis: O.P poisoning, post laparotomy ruptured ectopic pregnancy and type 1 respiratory failure.

CXR- wnl, same antibiotics and inj. Atropine and PAM were continued. Adequate oral and ETT suctioning were done. Patient was gradually weaned over the next 2 days.

Ivf-@ 70ml/hr RL, DNS, NS, U.0> 0.5ml/kg/min.

23/10/14- 11.30 am: Patient was well weaned to T-piece and maintained for $12 \mathrm{hrs}$.After fulfilling extubation criteria, patient was extubated. 02 inhalation by facemask @ 61/min.

ABG-ph-7.43, HC03-22, 02sat-99.9, pco2-34, po2-284, Chest X-ray- wnl, Sr. pseudocholinesterase- $3200 \mathrm{u} / \mathrm{l}, \mathrm{Hb} \%=10.4 \mathrm{gm} \%$, Muscle power $5 / 5$.

Patient was shifted to ICU on 25/10/14 and was continued on inj.atropine and PAM. Patient was shifted to AMC on 27/10/14 and discharged on 31/10/14.

DISCUSSION: Organophosphate poisoning results from exposure to organophosphates (OPs), which cause the inhibition of acetycholinesterase (AChE), leading to the accumulation of acetylcholine (ACh) in the body. Organophosphate poisoning most commonly results from exposure to insecticides or nerve agents. OPs are one of the most common causes of poisoning worldwide, and are frequently intentionally used in suicides in agrarian areas.[1,2] There are around 1 million OP poisonings per year with several hundred thousand resulting in fatalities annually.

Organophosphates inhibits, inactivates AChE. AChE is critical for nerve function, so the irreversible blockage of this enzyme, which causes acetylcholine accumulation, results in muscle over stimulation. This causes disturbances across the cholinergic synapses and can only be reactivated very slowly, sometimes not at all.

Overstimulation of nicotinic acetylcholine receptors in the central nervous system, due to accumulation of ACh, results in anxiety, headache, convulsions, ataxia, depression of respiration and circulation, tremor, general weakness, and potentially coma.

When there is expression of Muscarinic overstimulation due to excess acetylcholine at Muscarinic acetylcholine receptors symptoms of visual disturbances, tightness in chest, wheezing due to bronchoconstriction, increased bronchial secretions, increased salivation, lacrimation, sweating, peristalsis, and urination can occur.[3]

Even though all OP compounds have a common mechanism of action, their effectiveness as inhibitors of AChE, vary widely. OP compounds can be classified as direct or indirect inhibitors of AChE. Direct inhibitors are effective without any further metabolic modification after absorption into the body. Indirect inhibitors need to be transformed in the body to active form.

\section{The OP poisoning effects can be summarized as: ${ }^{[4]}$}

a) Muscarinic Effects: Salivation, Bronchorrhea, Bronchospasm, Bradycardia, Lacrimation, Diarrhea, Urination, G. I. distress, Emesis, Miosis, Excessive sweating.

b) Nicotinic Effects: Muscle Fasiculations, Cramping, Weakness, Diaphragmatic Paralysis, Respiratory Failure, Tachycardia and Hypertension. 


\section{CASE REPORT}

c) CNS effects: Headache, Tremors, restlessness, ataxia, confusion, slurred speech, seizures and coma are due to action on central nervous systems and at nicotinic receptors on skeletal muscle.

Once an organophosphate binds to AChE, the enzyme can undergo one of the Following: Endogenous hydrolysis of the phosphorylated enzyme by esterases or paraoxonases Reactivation by a strong nucleophile such as pralidoxime (2-PAM)

Three main syndromes can arise from OP poisoning in Humans: Acute cholinergic syndrome, Intermediate syndrome and OP-induced delayed polyneuropathy. The acute syndrome is treated with atropine, which blocks signalling through muscarinic acetylcholine receptors, thus acting as an antagonist of the parasympathetic nervous system.

However, in some cases muscle paralysis occurs24 to 96 hours after OP ingestion (intermediate syndrome), and this weakness, characteristic of IMS, may lead to respiratory failure and death.[5]

MECHANISM OF ACTION: (Irreversible binding and permanent enzyme inactivation (aging). The mechanism of action of OP compounds that has well been known for more than 70 years, is the inhibition of cholinesterase.[6]

\section{TWO TYPES OF CHOLINESTERASE ARE INVOLVED:}

1. Acetylcholinesterase (AChE), which is a specific enzyme for the diagnosis of OP poisoning and is called true cholinesterase. It is usually estimated in red blood cells (RBC) and is thus also called RBC ChE or erythrocyte acetylcholinesterase (EAChE).

2. Butyrylcholinesterase (BChE), which is less specific but more sensitive than AChE and is so called pseudocholinesterase. It is usually estimated in plasma and is thus also called plasma Ch E.

\section{The reaction between OP compounds and AChE occurs in three step reactions are:}

Step 1 is the formation of a reversible enzyme-inhibitor complex.

Step 2 is the phosphorylation and inactivation of the enzyme molecule.

Step 3 is the aging reaction involving formation of a monophosphoric acid residue bound to the enzyme.[10]

DIAGNOSIS: Blood, metabolites of cholinesterases, such as butyrylcholinesterase (BuChE) activity in plasma, neuropathy target esterase (NTE) in lymphocytes, and of acetylcholinesterase (AChE) activity in red blood cells Due to both AChE and BuChE being the main targets of organophosphates, their measurement is widely used as an indication of an exposure to an OP.[7]

The patients with OP poisoning can be divided into four groups of mild, moderate, severe, and fatal. 


\begin{tabular}{|l|l|l|}
\hline \multicolumn{1}{|c|}{ MILD } & \multicolumn{1}{|c|}{ MODERATE } & \multicolumn{1}{c|}{ SEVERE } \\
\hline Walks and talks headache, & Cannot walk & Unconscious, no papillary reflex. \\
dizzy & Soft voice & Muscle twitching, flaccid paralysis. \\
Abdominal pain & muscle & Increased bronchial secretions. \\
Sweating, salivation & twitching(fasciculations) & Dyspnoea crackles / wheeze. \\
Rhinorrhoea & Anxiety, restlessness & Possible convulsions \\
serum acetylcholinesterase & Small pupils (miosis) & Respiratory failure \\
enzyme (AChe) & serum acetylcholinesterase & serum acetylcholinesterase enzyme \\
Results: 1.6-4.0 u/ & enzyme (AChe) & (AChe)Results: < 0.8 u/ \\
\hline \multicolumn{2}{|c|}{ Table 1: Grading Severity of Organophosphate Poisoning[8] normal serum } \\
\hline \multicolumn{2}{|c|}{ acetycholinesterase / RBC Cholinesterase level is 8.0-20. } \\
\hline
\end{tabular}

\section{TREATMENT:[8]}

\section{FIRST AID:}

1. Decontamination, Assess breathing and circulation, Resuscitate if necessary, Support vital functions if necessary: oxygen therapy, lung ventilation, inotropes, Control of convulsions.

2. Monitor: ECG, blood pressure, oxygen saturation, ventilation, and level of consciousness.

\section{PREVENT FURTHER ABSORPTION OF INSECTICIDE:}

1. Gastric lavage: protect airway in patients with impaired consciousness,

2. Administer activated charcoal.

\section{INVESTIGATIONS:}

1. Arterial blood for oxygen and carbon dioxide partial pressures.

2. Venous blood for estimation of red cell acetylcholinesterase and concentration of the OP compound.

3. Venous blood for estimation of biochemistry (Electrolytes, glucose, amylase, lipase, creatinine) and haematology.

4. Chest X-ray.

5. Ultrasound scan of the abdomen (Pancreatic status).

Atropine or Glycopyrrolate[8]: Atropine acts as a physiological antidote, effectively antagonising the muscarinic receptor-mediated actions of excessive acetylcholine (increased tracheobronchial secretions, bradycardia, salivation, bronchoconstriction). Unlike glycopyrrolate, atropine crosses the blood-brain barrier and counteracts the effects of acetylcholine accumulation in the CNS that may cause convulsions and extra pyramidal symptoms.

The initial dose should be $2 \mathrm{mg}$ intravenously, repeated at 5-10 min intervals until signs of atropinisation occur: a pulse rate $>80$ beat. $\min ^{-1}$ and dilatation of the initially constricted pupils. Atropine therapy should be maintained until there is complete recovery. Atropine has been shown to enhance neuromuscular transmission and transmitter release, possibly by acting on muscarinic 
presynaptic inhibitory receptors, which are involved in the feedback mechanism of transmitter release. It has been shown that a smaller than traditional dose of atropine used over a shorter duration at a hospital with lower complication and mortality rates. A case of OP poisoning refractory to atropine, and in which glycopyrrolate was used to reduce cholinergic symptoms. ${ }^{[8]}$

Atropine must not be given until oxygenation is adequate, or ventricular fibrillation may occur.'

In order to obviate the added risk of hypoxia-induced ventricular dysrhythmias, correct cyanosis before administering atropine. Supplemental oxygen should be given, ideally before atropine administration, as hypoxia may increase the risk of atropine-induced dysrhythmias.'[9]

MAGNESIUM: Ventricular premature contractions were successfully eliminated with intravenous magnesium sulphate. The magnesium was thought to counteract the direct toxic inhibitory effect of OPs on sodium-potassium ATPase. An additional benefit in this setting may be magnesium's inhibitory effect on acetylcholine release, administered magnesium sulphate $4 \mathrm{~g}$ intravenously to patients intoxicated with OP and observed that the neuro-electrophysiological defects that had been observed earlier were reversed.[8]

OXIMES: Three actions have been attributed to pralidoxime,

1 reactivation of cholinesterase by cleavage of phospshorylated active sites; 2 direct reaction and detoxification of unbonded OP molecules; 3 endogenous anticholinergic effects in normal doses. Suggested therapeutic concentrations have been achieved for $6 \mathrm{~h}$ by intravenous administration of pralidoxime chloride (2-PAM) in 15-30 mg.kg-1 doses given intravenously. A continuous infusion with a dose of $550 \mathrm{mg} . \mathrm{h}^{-1}$ would provide similar therapeutic levels and has been advocated for severe poisoning. Pralidoxime mesylate (P2S) is less effective in producing sustained oxime concentrations and requires 4-hourly dosing, the balance between ageing and reactivation reaction rates for the inhibited acetylcholinesterase is altered in favour of the latter. Thus, benefit will accrue even if oxime therapy is commenced or continued several days after poisoning.[8]

DIAZEPAM: Some reports have indicated that benzodiazepines are potentially useful as antidotes to poisoning by cholinesterase inhibitors]. Diazepam has been useful in the management of convulsions after OP poisoning and in the support of ventilatory care.[8]

CLONIDINE: Clonidine inhibits the release of acetylcholine from central and peripheral cholinergic neurones. It was suggested that central cholinergic neurones involved in the regulation of respiration and fine motor control, but not peripheral motor neurones, are inhibited by the action of clonidine on $\alpha$ receptors. ${ }^{[8]}$

ANNEALED RED CELLS: Resealed cells containing a recombinant phosphotriesterase provided protection against the lethal effect of paraoxon. ${ }^{[8]}$ 


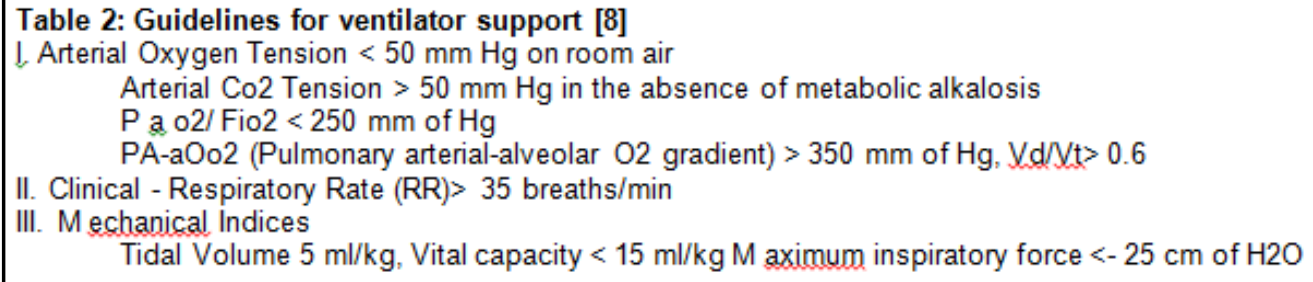

Organophosphorus poisoning and the Anaesthesia:[10] When a patient with organophosphorous compound poisoning comes for emergency surgery, the Anaesthesiologist should first carry out thorough examination. If anaesthesiologist feels that patient is not adequately treated then atropine and pralidoxime should be repeated till pupils in the mid position, secretions fully dried up, lungs clear and dry axilla.

Organophosphorus compounds provide the anaesthetist with demanding clinical challenges following acute exposure, during both the cholinergic phase and the intermediate syndrome. Occupational exposure to OP agents may manifest as nonspecific disorders in patients presenting for surgery. The effects of OP agents at the neuromuscular junction can alter the response to both depolarising and nondepolarising neuromuscular blockers. Altered neuromuscular function has been observed for up to 2 years after exposure. The activity of drugs hydrolysed by cholinesterase, e.g. local anaesthetics and esmolol, can be prolonged until total recovery of cholinesterase activity occurs. Disturbances in electrolyte balance, e.g. hypokalaemia, endocrine function, e.g. reduced adrenocorticotrophic hormone levels and hyperglycaemia, temperature regulation, immune responses, mental acuity and memory that could follow exposure to OP agents need to be considered during the conduct of an anaesthetic. Effects on the vocal cords are of particular relevance, as is the occurrence of pancreatitis and extrapyramidal disorders.

Unfortunately, there are no studies on responses to neuromuscular blocking agents during the intermediate syndrome, although a marked increase in sensitivity to nondepolarising neuromuscular blockers may be anticipated. This would be similar to the increased sensitivity to nondepolarising neuromuscular blockers after recovery of depolarising block.

The increased sensitivity after depolarising block may be up to seven-fold and can occur between 3 and $4 \mathrm{~h}$ after suxamethonium block. Cardiovascular effects may be long-lasting and the risk of life-threatening arrhythmias provides challenging therapeutic scenarios during anaesthesia and in intensive care.

CONTRAINDICATIONS: Drugs like morphine, succinylcholine, theophylline, phenothiazine and reserpine are contraindicated. Adrenergic amines should be given only if there is specific indication, such as marked by hypotension. ${ }^{[5]}$

INTERMEDIATE SYNDROME: IMS was first described as a syndrome of muscular paralysis occurring in conscious patients 24-96 h following ingestion, after their acute cholinergic syndrome was treated with atropine. Muscle weakness affected predominantly the proximal limb muscles and those supplied by the cranial nerves. IMS was often associated with respiratory failure. More recent work suggest that IMS could occur before $24 \mathrm{~h}$ and even after $96 \mathrm{~h}$ The pathophysiology of IMS is not clearly understood but is generally believed to result from a persistent excess of acetylcholine (ACh) at the neuromuscular junction.[10,12] 
Recent studies have shown that intermediate syndrome is accompanied by the excretion of cholinesterase inhibitor metabolites in the urine and by severe depression in cholinesterase levels. It has been suggested that the condition may reflect the recirculation of lipid soluble cholinesterase inhibitors from body fat compartments or gastric fluids. (De Bleecker, Willems et al. 1992; De Bleecker, Van Den Neucker et al. 1993).

Incidence: Estimates are that $10-68 \%$ of those poisoned with organophosphorus agents will develop intermediate syndrome. (Leon, Pradilla et al. 1996; Karalliedde, Wheeler et al. 2000)

Although some authors have maintained that the syndrome only occurs after severe cases of acute toxicity, (De Bleecker, Van Den Neucker et al. 1993; Ray 1998; Kwong 2002) Khan et al. (2001) found that the syndrome occurred in $22 \%$ of those with mild poisoning and $17 \%$ of those with moderate poisoning. (Khan, Hemalatha et al. 2001)

Signs and Symptoms Typically Seen (Karalliedde and Senanayake 1989; Clark 2002; Erdman 2004): Karalliedde (Karalliedde and Senanayake 1989) first described the syndrome in 1987 and observed that, although clinical findings occurred in a delayed fashion, they were described as acute in onset.

\section{Signs and symptoms that were typically present Included:}

- Muscle weakness and paralysis of.

- Muscles enervated by cranial nerves.

- Different combinations of muscles enervated by cranial nerves III-VII and X were involved.

- Neck flexors.

- A constant feature, and one of the earliest signs, was marked weakness of the neck flexors and inability of patients to raise their heads off their pillows.

- Proximal limb muscle weakness.

- This most typically involved shoulder abductors and hip flexors.

- Respiratory muscles.

- Patients initially were anxious and restless from hypoxia. Those that could would try to sit up to breath. They were bathed in sweat and using all their accessory respiratory muscles.

- Deep tendon reflexes.

- Deep tendon reflexes were decreased or absent in most patients, although cases of hyperreflexia has sometimes been seen.

- Muscle fasciculations.

- Fasciculations are rare, although spasticity and dystonic reactions were occasionally observed.

- Muscarinic signs.

- Some authors have concluded that muscarinic signs do not occur in patients with the intermediate syndrome (although --- as noted above --- sweating, anxiety, and restlessness, attributed to hypoxia, were noted). However, De Bleecker et al. (1993) (De Bleecker, Van Den Neucker et al. 1993) observed in a prospective study that 6 out of 8 cases developed short relapses of muscarinic findings that were relieved by increasing the atropine dosage. (De Bleecker, Van Den Neucker et al. 1993).

- Loss of sensation.

- Loss of sensation was not observed. 
COMPLICATIONS: The neuromuscular effects can progress to frank paralysis with respiratory failure and death. Unfortunately, many cases are not diagnosed until significant respiratory insufficiency has developed. (Erdman 2004) Data from India implicates the syndrome as the main cause of morbidity and mortality from organophosphorus poisoning. (Khan, Hemalatha et al. 2001).

TREATMENT: If muscarinic findings occur, they appear to respond to an increase in atropine dose. Although De Bleecker et al. (1993) concluded that oxime treatment did not alter the course of the syndrome; the doses used may have been insufficient. It has been suggested that the syndrome may reflect inadequate oxime therapy. In any case, supportive treatment, including mechanical ventilation when needed, is very important. (Kwong 2002).

DISPOSITION: The risk of intermediate syndrome has important implications for patient management, because those who have apparently recovered from the acute cholinergic toxidrome may then suffer from acute respiratory failure or arrest 3-4 days later. Close monitoring and observation during this period is therefore warranted. (Benson, Tolo et al. 1992)

PROGNOSIS: If there has not been hypoxic damage, and if proper supportive care has been provided, survival can be expected in most cases. The condition usually resolves spontaneously within 1-2 weeks. (Karalliedde and Senanayake 1989; Erdman 2004) The intermediate syndrome is a condition of muscular weakness and paralysis that occurs 1-4 days after the resolution of acute cholinergic toxidrome due to organophosphate exposure.

Many cases are not diagnosed until significant respiratory insufficiency has occurred.

It can be a major cause of organophosphate-induced morbidity and mortality.

If hypoxic damage has not occurred, and with proper supportive care, the condition usually resolves spontaneously in 1-2 weeks

- Key Points: The intermediate syndrome is a condition of muscular weakness and paralysis that occurs 1-4 days after the resolution of acute cholinergic toxidrome due to organophosphate exposure.

- Many cases are not diagnosed until significant respiratory insufficiency has occurred.

- It can be a major cause of organophosphate-induced morbidity and mortality.

If hypoxic damage has not occurred, and with proper supportive care, the condition usually resolves spontaneously in 1-2 weeks.

The peripheral neuropathy after poisoning may become evident within two or five weeks. The low pseudo-cholinesterase levels may persist for 15 days to one month. So one should avoid succinylcholine during this period.[13]

CONCLUSION: However, history of exposure to organophosphorous compounds and clinical manifestations of a cholinergic syndrome are sufficient for management of the affected patients. The standard management of poisoning with organophosphorous compounds consists of decontamination, and injection of atropine sulfate with an oxime. Recent advances on treatment of organophosphorus pesticides poisoning revealed that blood alkalinization with sodium bicarbonate and also magnesium sulfate as adjunctive therapies are promising. Patients who receive prompt proper treatment usually recover from acute toxicity but may suffer from neurologic complications. 
Anaesthetic management of Organophosphorous pesticide poisoning demands close observation, timely institution of antidote in adequate doses and duration and good supportive care. Good understanding of acetyl choline and cholinesterase activity and drugs which are to be used and which are to be avoided is vital for successful management of the cases.

\section{REFERENCES:}

1. Pandit V, Seshadri S, Rao SN, Samarasinghe C, Kumar A, Valsalan R (Jan-Mar 2011)."A case of organophosphate poisoning presenting with seizure and unavailable history of parenteral suicide attempt". J Emerg Trauma Shock 4 (1): 132-4. doi:10.4103/0974-2700.76825. PMC 3097564. PMID 21633583.

2. Yurumez Y, Durukan P, Yavuz Y, Ikizceli I, Avsarogullari L, Ozkan S, Akdur O, Ozdemir C (2007)."Acute organophosphate poisoning in university hospital emergency room patients". Intern Med 46 (13): 965-9. doi:10.2169/internalmedicine.46.6304. PMID 17603234.

3. Leibson T, Lifshitz M (2008)."Organophosphate and Carbamate Poisoning: Review of the Current Literature and Summary of Clinical and Laboratory Experience in Southern Israel". J Toxicology 10: 767-7704.

4. KRUPESH, CHANDRASHEKAR, ASHOK,: ORGANOPHOSPHORUS POISONING, Indian J. Anaesth. 2002; 46 (1): 40-43.

5. Jayawardane, P., Dawson, A.H., Weerasinghe, V., Karalliedde, L., Buckley, N.A., Senanayake, N., Bateman, N. (2008). The Spectrum of Intermediate Syndrome Following Acute Organophosphate Poisoning: A Prospective Cohort Study from Sri Lanka. PLoS Medicine, 5(7), e147. DOI: 10.1371/journal. pmed. 0050147.

6. Mahdi Balali-Mood MD PhD*, Kia Balali-Mood PhD** Neurotoxic Disorders of Organophosphorus Compounds and Their Managements Archives of Iranian Medicine, Volume 11, Number 1, 2008: 65 - 89.

7. Worek F, Koller M, Thiermann H, Szinicz L (2005).”Diagnostic aspects of organophosphate poisoning". J Toxicology 214: 182-9.

8. L. Karalliedde, Organophosphorus poisoning and anaesthesia Article first published online: 6 APR 2002,DOI: 10.1046/j.1365-2044.1999.01061

9. L. A. KONICKX, 1,2 K. BINGHAM, 1 and M. EDDLESTON 1,3 Is oxygen required before atropine administration in organophosphorus or carbamate pesticide poisoning? -A cohort studyClin Toxicol (Phila). Jun 2014; 52(5): 531-537

10. NK Sundaray, Ratheesh Kumar 13 May 2009. Organophosphorous Poisoning: Current management guidelines. 421 in stool locally. Update in Anaesthesia 2005; 19:1-9. 22.

11. Khan NU, Patel MJ, Hifath MNM, Kamal S, Hussain SA (2012) Prolonged Intermediate Syndrome Due to Organophosphate Poisoning. 2: 229. doi:10.4172/scientificreports.229. Published online May 8, 2014. doi: 10.3109/15563650.2014.915411PMCID: PMC4134047.

12. Karalliedde L ${ }^{1}$, Baker D, Marrs TC. Organophosphate-induced intermediate syndrome: aetiology and relationships with myopathy See comment in PubMed Commons below Toxicol Rev. 2006; 25 (1): 1-14.

13. Agency for Toxic Substances and Disease Registry: - Course: WB 1098- Environmental Health and Medicine Education-Cholinesterase Inhibitors: Including Insecticides and Chemical Warfare Nerve Agents. Part 5: The Intermediate Syndrome- Page-104 to 106. 


\section{AUTHORS:}

1. N. Gopal Reddy

2. Kahlekar

3. Laxmi Priyanka

\section{PARTICULARS OF CONTRIBUTORS:}

1. Associate Professor, Department of Anaesthiology, KIMS, NKP.

2. Assistant Professor, Department of Anaesthiology, KIMS, NKP.

3. Post Graduate, Department of Anaesthiology, KIMS, NKP.

\section{NAME ADDRESS EMAIL ID OF THE} CORRESPONDING AUTHOR:

Dr. N. Gopal Reddy, Plot No. 54, Old V. T. Colony, Nalgonda-508001,

Telangana State, India.

E-mail: drgopalreddynarra@yahoo.com

Date of Submission: 11/12/2014. Date of Peer Review: 12/12/2014. Date of Acceptance: 29/12/2014. Date of Publishing: 05/01/2015. 